\title{
Research on Enterprise Internal Control Based on Accounting Computerization
}

\author{
Jun Feng \\ Business Administration College, Wuhan Business University, Wuhan Hubei, 430056, China
}

Keywords: Accounting computerization, Enterprise internal control, Control management

\begin{abstract}
As economic development level in China keeps improving, accounting computerization has achieved rapid development and progress. With continuous development and widespread application of computer technology, accounting computerization becomes a development trend of accounting work. Accounting computerization work involves numerous contents, including system development, implementation control, management control, and routine control management etc. The problems of accounting computerization implementation can be found through analysis of these contents, such as original data accuracy, operating control problem, paperless accounting file and intangible operation. This paper will research and analyze enterprise internal control from the perspective of accounting computerization to make sure accounting computerization system operation is safer and more effective and the data are more authentic and reliable.
\end{abstract}

\section{Introduction}

Enterprise internal control not merely involves enterprise property, material security and integrity, but also directly reflects and embodies enterprises' economic activity. To achieve development goal, enterprises should establish internal control system and ensure sustainable and sound development. After an enterprise establishes computerization system, numerous changes occur in financial accounting and management method. Accounting computerization becomes computer-based accounting practice, and can keep accounts, cast accounts, report accounts and inquire accounts based on computation. This alters the situation of computation, statistics and analysis by human brain, and makes the work conducted in a more timely, efficient and rapid way. Compared with original manual accounting, accounting computerization has a lot of advantages, such as more timely and rapid data sharing and retrieval and easier statement preparation and modification. In specific work, accounting computerization can greatly save human power, material resources and time. Meanwhile, accounting computerization also brings new problems for enterprise internal control. If these problems cannot be discovered and handled in time, orderly development of enterprises will be hindered.

\section{Problems of accounting computerization in enterprise internal control}

As information technology continuously develops, enterprises' application of information technology has become very widespread. With the emergence and development of information technology, accounting computerization has the development history of over 10 years. After 1990s, computer technology developed faster. Computerized accounting software has changed to systematized numerical accounting and statistics from simple numerical calculation, and develops to artificial intelligence field. Even if accounting computerization has achieved many results in China, some problems still exist.

\section{Low original data operation accuracy}

In application of accounting computerization, computer operation can automatically achieve data output or input, store or process the inputted data. The data are mainly stored in magnetic media. Besides, automatic account keeping, analysis and accounting statement preparation can be conducted under computer process control. But, original data in the computer must be accounted manually. After they are free from error, they can be inputted in the computer. If original data input goes wrong, it will be difficult for the computer to recognize the data. The computed data are also wrong, which 
will influence follow-up work. This defect of accounting computerization directly puts forward higher requirements for internal control: it is required to standardize data processing method to make them more accurate and stable, improve accounting information quality and keep integrity and accuracy $^{[1]}$.

\section{Duty dereliction of control operators}

Usually, enterprise internal control involves authorization and approval control. In manual accounting operation, the persons with corresponding right should sign and check each basic link for some economic business so as to reduce errors. The steps are tedious and troublesome. The application of accounting computerization leads to fundamental changes of various enterprise businesses. Different businesses are dominated by computerization so that computerization functions and knowledge are concentrated. Such highly centralized state makes the functions of accounting personnel change to management-based talents from accounting-based talents. Some personnel alter their work to data submission and preparation from data input. Thus, if enterprise internal control cannot be done well, computer operators will directly modify system program or database and manipulate the results. Thus, fraud risk will occur easily ${ }^{[2]}$.

\section{Paperless accounting file and intangible computer operation}

In the past manual method, all kinds of data information are stored in different paper in the form of accounts, statement and certificate. The personnel just need to tick on the account book for the increase or decrease. As time goes, many handwritings and seals are disordered. Others are difficult to see clearly. After computerized accounting is adopted, traditional recording method decreases a lot, and manual accounting system is replaced by tangible records. Many accounting operations including information entry, reading or query will be operated in computation. Besides, data carrier also alters. Data and program become information sources. Disk medium is used for storage. Some businesses or handling results can be directly printed, and the computer needs to be used to inquire these data. If an enterprise is unable to carry out effective internal control or managers alter data at will, the useful data may not be found, and data loss will be caused. Besides, data alteration has no trace. The damage to electronic disk medium or internal data loss will result in crisis to the enterprise. Thus, accounting data or other data need both paper storage method and computer disk storage to enhance protection of documents and data, improve internal control and promote computerization progress ${ }^{[3]}$.

\section{Security problem in accounting computerization work}

a). At present, many accounting software systems have such defects as small demand and few functions in design, development or application. The language or database of software originally designed has low security. Once the software is put into use, many security problems will be exposed. For example, the database is under open state for a long time; it is easier to open and seek the data and document; the application system also has many security loopholes. Besides, the above problems cannot be completely eradicated. Meanwhile, security protection facilities and equipment are not sound, and hardware and software have poor properties, these directly influence system software operation. Moreover, some operators or managers are short of security awareness, and fail to set security code in time. Management system is not sound, and inspection is not timely.

b). As information technology develops continuously, information system networking is also faced with many security problems as follows: internet is based on INTER NET/INTRANET system; the protocol used is TCP/IP; broadcast mode is selected for communication; it is easier to intercept, filch password and filch. E-commerce embodies network economy and involves numerous contents, such as electronic billing and electronic money. It offers conditions for lawbreakers' hostile attack ${ }^{[4]}$.

c). Accounting informationization will promote financial management and businessmanagement to form integration trend. Various operation activities of an enterprise greatly depend on network system. If enterprise network management and maintenance level is too low or no monitoring is adopted, system paralysis will be caused easily, and enterprise production and operation will be affected. Thus, it is very critical for enterprises to conduct internal control well. 


\section{Internal control countermeasures under accounting computerization}

\section{To enhance program operation control}

To better process information and prevent serious accidents or deviation, it is required to formulate and improve principal computer rules and operation regulations, and embody operating control system. Principal computer rules are a part of internal management of computer room, while operation regulations are the steps and requirements proposed for computer service, including operation industrious, equipment use and personnel operation specifications etc. To be more specific, operation regulations involves stringent examination, approval and entry of data, integrity and authenticity of original certificate; if data entry goes wrong and timely checking and entry are required, one should report to the superior and should not modify or delete at will; except the personnel in the computer room, external persons should be guarded strictly to prevent data modification or stealing; external persons should not be taken to the computer room at will. In case of wrong data information, modification should be conducted under the system instruction, such as supplementary registration requires correction of the certificate. After formal operation, operators shall not leave from their posts at will, and rotating shift system should be adopted. Important data and information should be backed up weekly, monthly and quarterly. Once the system forms, it can continue to be improved according to actual conditions of enterprise operation. The error rate can be minimized and authenticity and integrity of accounting information can be ensured only when an enterprise formulates complete, reliable and specific system.

\section{To enhance control of personnel functions}

Organizational control is to segment irrelevant duties in the system, divide the work for personnel at all levels, formulate supporting management rules according to the functions and make functional management more efficient and feasible. The purpose of functional control is to establish a mechanism with mutual supervision, auditing and dependence so as to ensure reliable, secure and real information and prevent favoritism and irregularities. The most important point of division of duties is to distinguish the duties of computerization department and user department. User department is original data source department. The work of these departments is to gather and sort data. The duties are divided among the two departments to independently complete authorization, execution, preservation and recording and to separate internal duties of computerization department. Division of internal duties can prevent high concentration of functions and make up for non-uniform functions or shortcomings of centralization. To control operators' functions in accounting computerization, it is required to divide functions according to different software supported by the system. In this way, the right of each department can be checked and balanced. For instance, commercial enterprises use LAN server, PC machine and POS machine for software control. They start from their own features and establish favorable confidentiality mechanism in accordance with ORACLE8 database. Besides, ORACLE8 ("backstage") which operates on SUN server can be set in combination of password technology. Then, LINIX operating system and POS machine are used to achieve data input or output. Procurement link needs registration first, and then the finance department audits data and finally records them in the account. The purpose of various statements is for account records. Thus, different departments check and supervise each other ${ }^{[5]}$.

\section{To establish and improve file management system}

Enterprise internal file mostly refers to various account books, accounting statements, certificates, bills and disk media etc. The use of these contents must be managed. The system develops file data and accounting data so as to ensure file integrity, preservation and confidentiality in the system. Besides, file management formulation and implementation generally meet the following aspects: the accountant officer and system administrator are responsible for signing and sealing, and then the files can be saved. It is required to implement effective protection measures; the backup soft and hard disks can be labeled, numbered and placed where it is dry and ventilated. If disk medium is used to save accounting file, regular inspection and duplication should be conducted to check whether the 
contents in the disk are integral and real. In addition, talent training should be valued, and regular education and training should be conducted to improve employees' professional quality. Meanwhile, the accounting team with high quality and strong professional ability should be built to support accounting computerization.

\section{To enhance system internal control}

Enterprise internal control aims to guarantee computer system operation security and stability and prevent external interference and wrong operation. Security control mainly includes the following aspects: contact control, environmental protection, security control and security management etc. Contact control is to prevent non-authorized persons from using system resources at will and ensure internal resource correctness. The control contents include the following: formulate internal operation system, and prevent non-authorized persons from operating the computer; set operating authorization restriction; adopt identity password control for operators; segregate data storage and processing; set contact and operation log control. The above control aims to reduce external interference of computer operation, decrease computer operation fault, and guarantee secure and stable computer operation. In addition, environmental protection, equipment allocation and power supply system installation are also involved.

Finally, internal audit should be enhanced. Internal audit is an important component of internal control, and aims to intensify internal accounting supervision system. In accounting computerization, since "human-machine" dialogue has special form, more standards and requirements are put forward for internal audit. Internal audit involves the following contents: regularly audit accounting data, investigate accounting computerization account, check whether it is correct and strictly follows standards in Accounting Law. The cost signature should adapt to internal control system. It is required to investigate the vouchers, analyze whether they are integral and standard; computer internal data and written data consistency should be examined, e.g. whether account content conforms to statement content, and adjust wrong accounts in time; supervise data preservation process, and analyze whether data preservation is legal and secure to prevent illegal data modification; enhance investigation of each system operation link to prevent examination loophole or fault.

\section{Conclusion}

This paper mainly analyzes problems in enterprise internal control from the perspective of accounting computerization and proposes several countermeasures to these problems. Enterprise internal control concerns future development of an enterprise, while accounting computerization is also an important content. The two influence each other and are dispensable. Therefore, it is very critical to enhance internal control from the perspective of accounting computerization. It is also the key to achieving secure and stable development of an enterprise.

\section{References}

[1] Hu Jie, Zhang Xuying, Jiang Cui et al., Study on Problems and Countermeasures of Enterprise Internal Control under Accounting Computerization. Commercial Accounting, 2012(12):118-119.

[2] Cui Bin, Zhang Ying, Research on Accounting Computerization Management in Farmers' Professional Cooperative Development - Case Study of Jiangsu Province. Jiangsu Agricultural Sciences, 2011,39(6):654-657.

[3] Ouyang Qin, Accounting Computerization Teaching Reform under Information Technology-Effective Combination of Online and Offline Study. Commercial Accounting, 2015(22):128-129.

[4] Du Xiaotong, Influence Factor Analysis of Accounting Computerization Carried out by Middle and Small-sized Enterprises and Suggestions. Commercial Accounting, 2013(24):109-110. 
[5] Zhao Xia, Study on Problems in Enterprise Internal Control under Accounting Computerization. China Business \& Trade, 2014(29):62-63. 